\title{
Long Noncoding RNA, ANRIL, Regulates the Proliferation of Head and Neck Squamous Cell Carcinoma
}

\author{
NATSUMI MATSUNAGA ${ }^{1}$, TAKAHIRO WAKASAKI ${ }^{2}$, RYUJI YASUMATSU ${ }^{2}$ and YOJIRO KOTAKE KA $^{1,3}$ \\ ${ }^{1}$ Graduate School of Humanity-Oriented Science and Engineering, Kindai University, Fukuoka, Japan; \\ ${ }^{2}$ Department of Otorhinolaryngology, Graduate School of Medical Sciences, Kyushu University, Fukuoka, Japan; \\ ${ }^{3}$ Department of Biological and Environmental Chemistry, \\ Faculty of Humanity-Oriented Science and Engineering, Kindai University, Fukuoka, Japan
}

\begin{abstract}
Background/Aim: ANRIL is a long noncoding RNA located on INK4 locus, which encodes p15 and p16 that cause $G_{1}$ phase arrest in the cell cycle. ANRIL positively regulates proliferation of several kinds of cancer cells such as lung and gastric cancers. This study, examined the effect of ANRIL in head and neck squamous cell carcinoma cells. Materials and Methods: Cells were transfected with siRNA oligonucleotides targeting ANRIL. Transfected cells were subjected to cell-cycle and quantitative reverse transcriptionpolymerase chain reaction ( $q R T-P C R)$ analysis. Results: Depletion of ANRIL increased p15 mRNA in FaDu cells, and p15 and p16 mRNA in CAL27 cells and inhibited proliferation of these cells. Cell cycle analysis showed that depletion of ANRIL caused arrest at the $G_{1}$ phase of the cell cycle. Conclusion: ANRIL promotes $G_{1}$ phase progression by repressing $p 15$ and p16, and thus promotes $F a D u$ and CAL27 cell proliferation.
\end{abstract}

INK4 locus (inhibitor of cyclin dependent kinase 4 locus) is located in the human chromosome 9p21 region. This locus encodes $p 15, p 16$, and $A R F$ genes, products of which function as tumor suppressors. Both p15 and p16 are CDK inhibitors that cause $G_{1}$ phase arrest in the cell cycle via inhibition of the CDK4 and CDK6 kinase activities (1). ARF functions to stabilize tumor suppressor p53 at the protein level by inhibiting the ubiquitin ligase activity of MDM2 (2, $3)$. This locus is frequently either mutated, deleted, or its transcription is repressed in many types of human cancers

Correspondence to: Yojiro Kotake, Ph.D., Department of Biological and Environmental Chemistry, Faculty of Humanity-Oriented Science and Engineering, Kindai University, 11-6 Kayanomori, Iizuka, Fukuoka 820-8555, Japan. Tel: +81 0948225659 (469), Fax: +810948230536, e-mail: ykotake@fuk.kindai.ac.jp

Key Words: ANRIL, CDK inhibitor, head and neck squamous cell carcinoma, cell cycle.
(4). The transcriptional activation of this locus is therefore thought to be important in tumor suppression.

Long noncoding RNAs (lncRNAs) are more than 200 nucleotides long and lack a functional open reading frame. Recent studies have revealed that many lncRNAs participate in the regulation of differentiation, growth, senescence, and apoptosis (5). An antisense noncoding RNA transcribed from the INK4 locus (ANRIL) is the lncRNA (6). Along with Yap et al., we revealed that $A N R I L$ associates with polycomb repression complex 1 (PRC1) and 2 (PRC2) and recruits them on the INK4 locus to repress the transcription of $p 15$ and $p 16$ genes $(7,8)$. In the gene silencing model of polycomb protein complexes, PRC2 methylates histone $\mathrm{H} 3$ at lysine 27 on the target region, leading to the recruitment of PRC1, which ubiquitinates histone $\mathrm{H} 2 \mathrm{~A}$ at lysine 119 (9-11). These histone modifications establish and maintain the epigenetic repression of target genes. Silencing ANRIL results in the induction of $p 15$ and $p 16$ transcription, thus inhibiting cell proliferation $(7,8)$. Other than $p 15$ and $p 16$, ANRIL is involved in the transcriptional regulation of many genes related to cell adhesion, gene expression and apoptosis (12-14), indicating that ANRIL participates in the regulation of diverse cellular functions.

Expression analysis showed that ANRIL is expressed at higher levels in several human cancer cells, including human cervical cancer, osteosarcoma and non-small cell lung cancer than normal lung fibroblasts (15). ANRIL regulates cancer cell proliferation in an INK4 locus-dependent (15) and independent manner (16). Many studies on clinical cancer specimens have shown that the expression of ANRIL is upregulated in various human cancers such as lung, gastric, ovarian and cervical cancers (17). It has also been reported very recently that $A N R I L$ expression is up-regulated in oral cancer and that ANRIL participates in the proliferation, migration and invasion of oral squamous cell carcinoma cells (18). However, the role of ANRIL in head and neck squamous cell carcinoma is largely unknown. In this study, we examined the function and mechanism of action of ANRIL in head and neck squamous cell carcinoma cells. 


\section{Materials and Methods}

Cell culture and RNA interference. A human hypopharyngeal cancer cell line, FaDu and a human oral cancer cell line, CAL27 (American Type Culture Collection, Frederick, MD, USA), were cultured in Dulbecco's modified Eagle's medium (Gibco, Grand Island, NY, USA) supplemented with $10 \%$ fetal bovine serum (Gibco) at $37^{\circ} \mathrm{C}$ in an atmosphere containing $5 \% \mathrm{CO}_{2}$. For ANRIL silencing, siRNA oligonucleotides targeting ANRIL were transfected by using Lipofectamine RNAiMAX according to the manufacturer's instructions (Thermo Fisher Scientific, Waltham, MA, USA). The nucleotide sequence of the ANRIL siRNA oligonucleotide was 5'GGUCAUCUCAUUGCUCUAU-3' with 3' dTdT overhangs.

Quantitative reverse transcription polymerase chain reaction ( $q R T$ $P C R$ ). Seventy-two h after transfection of siRNA oligonucleotides, cells were subjected to qRT-PCR. qRT-PCR was performed as previously described (19). Briefly, the isolation of total RNA was performed using RNeasy Plus kit (Qiagen, Hilden, Germany). cDNA was synthesized using the SuperScript III First-Strand Synthesis System (Thermo Fisher Scientific) and amplified using QuantiTect SYBR Green PCR kit (Qiagen). The specific primer set for ANRIL was 5'-CTATCCGCCAATCAGGAGGC-3' and 5'GCGGCAGCGGTTTAGTTTAAT -3'. The specific primer sets for $p 15, p 16, A R F$ and $G A P D H$ have been previously described (15).

Cell cycle analysis. Cell cycle analysis was performed as previously described (20). Briefly, cells were fixed for at least $3 \mathrm{~h}$ at $-20^{\circ} \mathrm{C}$ using $70 \%$ ethanol about $72 \mathrm{~h}$ after transfecting siRNA oligonucleotides. The fixed cells were processed with Muse ${ }^{\mathrm{TM}}$ Cell Cycle Kit (Merck Millipore, Darmstadt, Germany) according to the instructions of the manufacturer and subjected to cell cycle analysis using Muse Cell Analyzer (Merck Millipore).

Statistical analysis. Data are presented as means and standard deviations from triplicate samples. A two-tailed $t$-test was performed to determine significant differences. A $p$-values of $<0.05$ was considered to be statistically significant.

\section{Results}

We had previously reported that ANRIL is involved in the repression of cellular senescence in normal lung fibroblasts (8). Recently, several other research groups, including ours, reported that $A N R I L$ functions to promote the proliferation of cancer cells, such as colorectal cancer, lung cancer, cervical cancer, gastric cancer, hepatocellular carcinoma and bladder cancer cells (17). Accordingly, we first examined the involvement of ANRIL in head and neck squamous cell carcinoma cell proliferation. The expression of ANRIL was downregulated using siRNA oligonucleotides that target $A N R I L$ in a human hypopharyngeal cancer cell line, $\mathrm{FaDu}$, and a human oral cancer cell line, CAL27. Depletion of ANRIL significantly repressed the proliferation of both $\mathrm{FaDu}$ (Figure 1A and B) and CAL27 (Figure 1C and D) cells, suggesting that ANRIL functions to promote the proliferation of both FaDu and CAL27 cells.
We (8), along with Yap et al. (7) have previously shown that ANRIL inhibits $p 15$ and p16 expression, thus resulting in the promotion of cell proliferation. Accordingly, we examined the involvement of ANRIL in p15 and p16 expression in $\mathrm{FaDu}$ and CAL27 cells. Data obtained from qRT-PCR demonstrated that transfection with siRNA oligonucleotides significantly reduced $A N R I L$ expression (Figure $2 \mathrm{~A}$ and $\mathrm{B}$ ) in both $\mathrm{FaDu}$ and CAL27 cells. Depletion of ANRIL resulted in an increase in $p 15$ mRNA expression in FaDu cells (p16 was not detected) (Figure 2A). Interestingly, silencing ANRIL also increased the expression levels of ARF mRNA that is transcribed from the INK4 locus and functions to stabilize the p53 tumor suppressor protein. Depletion of ANRIL resulted in an increase in $p 15$, p16 and ARF mRNA expression levels in CAL27 cells (Figure $2 \mathrm{~B})$. These results suggest that $A N R I L$ is also involved in the repression of $p 15$ and $A R F$ transcription in FaDu cells, and p15, p16 and ARF in CAL27 cells.

Given that depletion of ANRIL increased the mRNA levels of $p 15$ and $p 16$, both of which cause arrest at the $\mathrm{G}_{1}$ phase of the cell cycle, we next examined whether depletion of ANRIL affects the cell cycle in FaDu and CAL27 cells. Cell cycle analysis showed that depletion of ANRIL significantly reduced the number of cells in $S$ and $G_{2} / M$ phase and increased the number of cells in the $\mathrm{G}_{1}$ phase in both $\mathrm{FaDu}$ (Figure 3A and B) and CAL27 (Figure 3C and D) cells. This result suggests that $A N R I L$ is involved in the regulation of $\mathrm{G}_{1}$ phase progression in FaDu and CAL27 cells.

\section{Discussion}

Recent studies have shown that ANRIL induces proliferation of several cancer cells by regulating gene expression (15-17). In this study, we also showed that silencing ANRIL inhibits the proliferation of head and neck squamous cell carcinoma cells such as the human hypopharyngeal cancer cell line, FaDu and the human oral cancer cell line, CAL27, indicating that ANRIL functions as a positive regulator of proliferation of these cells. Furthermore, silencing ANRIL increased the expression of CDK inhibitors $p 15$ and $p 16$ on the INK4 locus, which lead to $\mathrm{G}_{1}$ phase arrest in the cell cycle in FaDu and CAL27 cells, suggesting that ANRIL functions to promote $\mathrm{G}_{1}-\mathrm{S}$ transition via repression of $p 15$ and $p 16$. ANRIL is known to repress $p 15$ and p16 transcription through the recruitment of polycomb complexes PRC1 and PRC2 on INK4 locus $(7,8)$. ANRIL also participates in the regulation of cell adhesion, apoptosis and metabolic activity via regulation of the transcription of many genes, in a trans-acting manner (14). The roles of ANRIL in processes other than cell cycle regulation and in target genes other than those of the INK4 locus are yet to be determined in head and neck squamous cell carcinoma and are important topics that warrant further study.

Many studies have shown that ANRIL expression is upregulated in various human cancers such as lung, gastric, 


\section{A \\ FaDu cells}
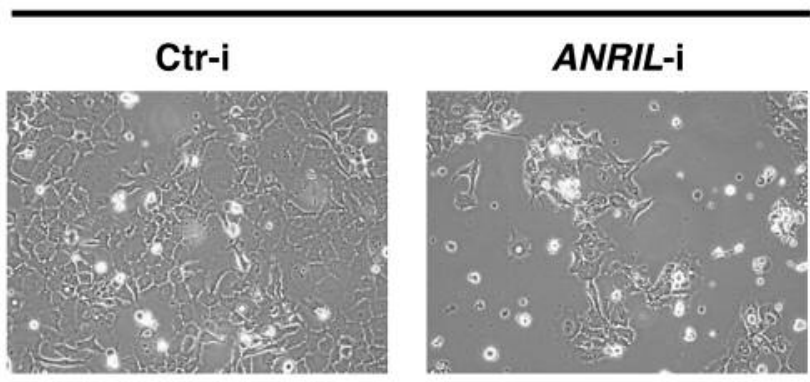

C

CAL27 cells
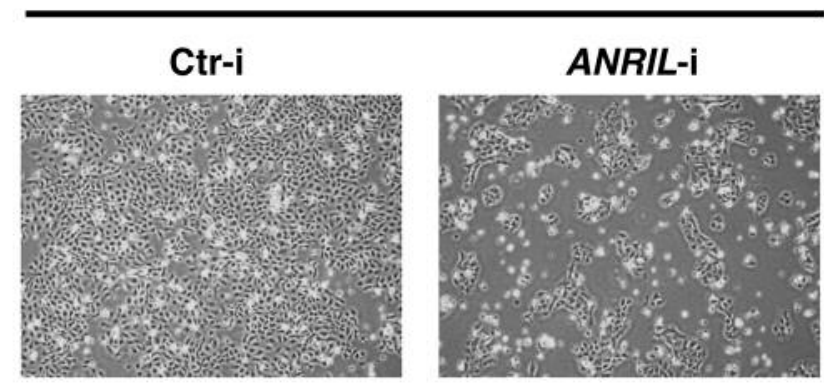
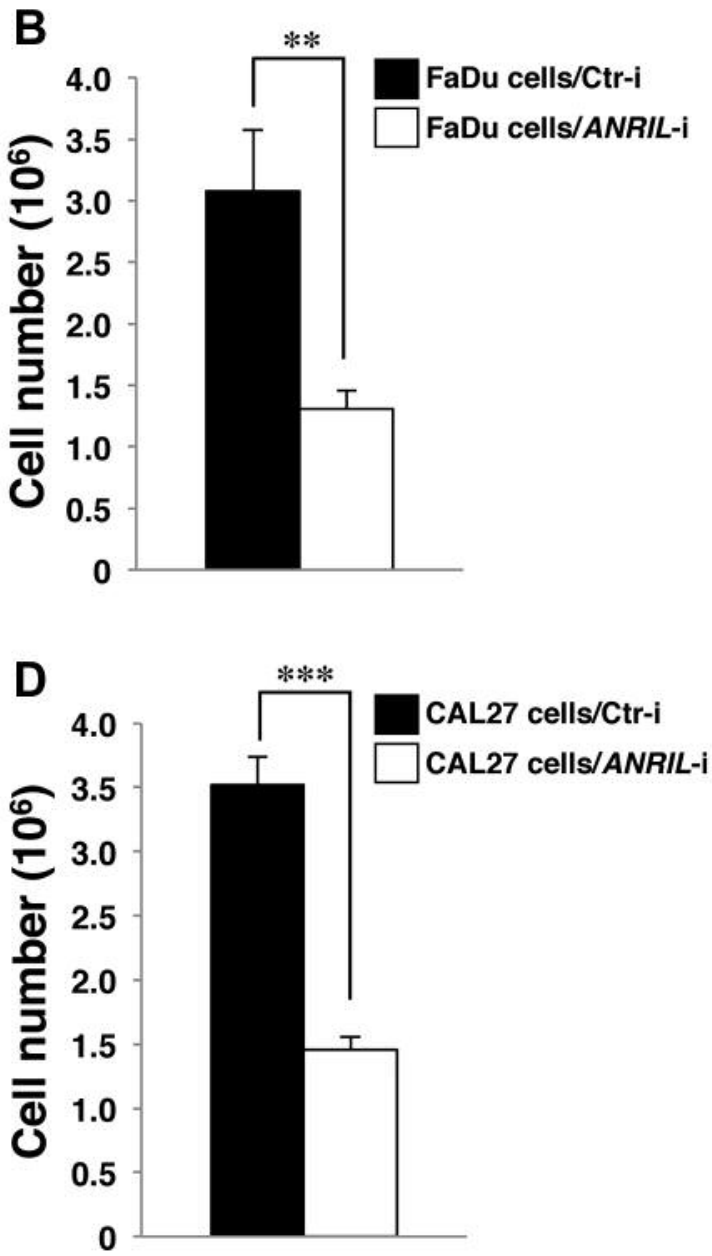

Figure 1. Depletion of ANRIL inhibits proliferation of FaDu and CAL27 cells. A: FaDu cells were seeded at $5 \times 10^{5}$ cells/100mm dish. One day later, the cells were transfected with either control siRNA (Ctr-i) or siRNA silencing ANRIL (ANRIL-i) and 72 h later, cells were photographed. B: The number of viable cells was measured by trypan blue staining. ${ }^{* *} p<0.01$. C: CAL27 cells were transfected with siRNA and observed as in (A). $D$ : Viable CAL27 cells were counted as in $(B) . *^{* *} p<0.001$.

ovarian and cervical cancers (17). It has also been reported very recently that ANRIL is highly expressed in head and neck squamous cell carcinoma and correlates with tumor progression (21). Accordingly, we propose that ANRIL upregulation causes aberrant progression of the cell cycle via repressing p15 and p16 transcription, resulting in head and neck squamous cell carcinoma progression.

\section{Conflicts of Interest}

The Authors have no conflicts of interest directly relevant to the contents of this article.

\section{Authors' Contributions}

Conceptualization and design, N.M., T.W., R.Y., and Y.K.; Supervision, Y.K.; Materials, N.M., T.W., R.Y., and Y.K.; Data collection, N.M., and Y.K.; Analysis, N.M., and Y.K.; Writer; Y.K.

\section{Acknowledgements}

The Authors would like to thank Hiroki Tsujioka, Takeshi Tsuruda, Kousei Nagayasu, Seiki U, Takazumi Uenishi and Ryotaro Okada for their helpful discussions and technical assistance. This work was supported by JSPS KAKENHI Grant Number 17K07184 (to YK) and the Naito Foundation (to YK).

\section{References}

1 Pei XH and Xiong Y: Biochemical and cellular mechanisms of mammalian CDK inhibitors: a few unresolved issues. Oncogene 24: 2787-2795, 2005. PMID: 15838515. DOI: 10.1038/ sj.onc. 1208611

2 Pomerantz J, Schreiber-Agus N, Liegeois NJ, Silverman A, Alland L, Chin L, Potes J, Chen K, Orlow I, Lee HW, CordonCardo C and DePinho RA: The Ink4a tumor suppressor gene product, p19Arf, interacts with MDM2 and neutralizes MDM2's inhibition of p53. Cell 92: 713-723, 1998. PMID: 9529248. 

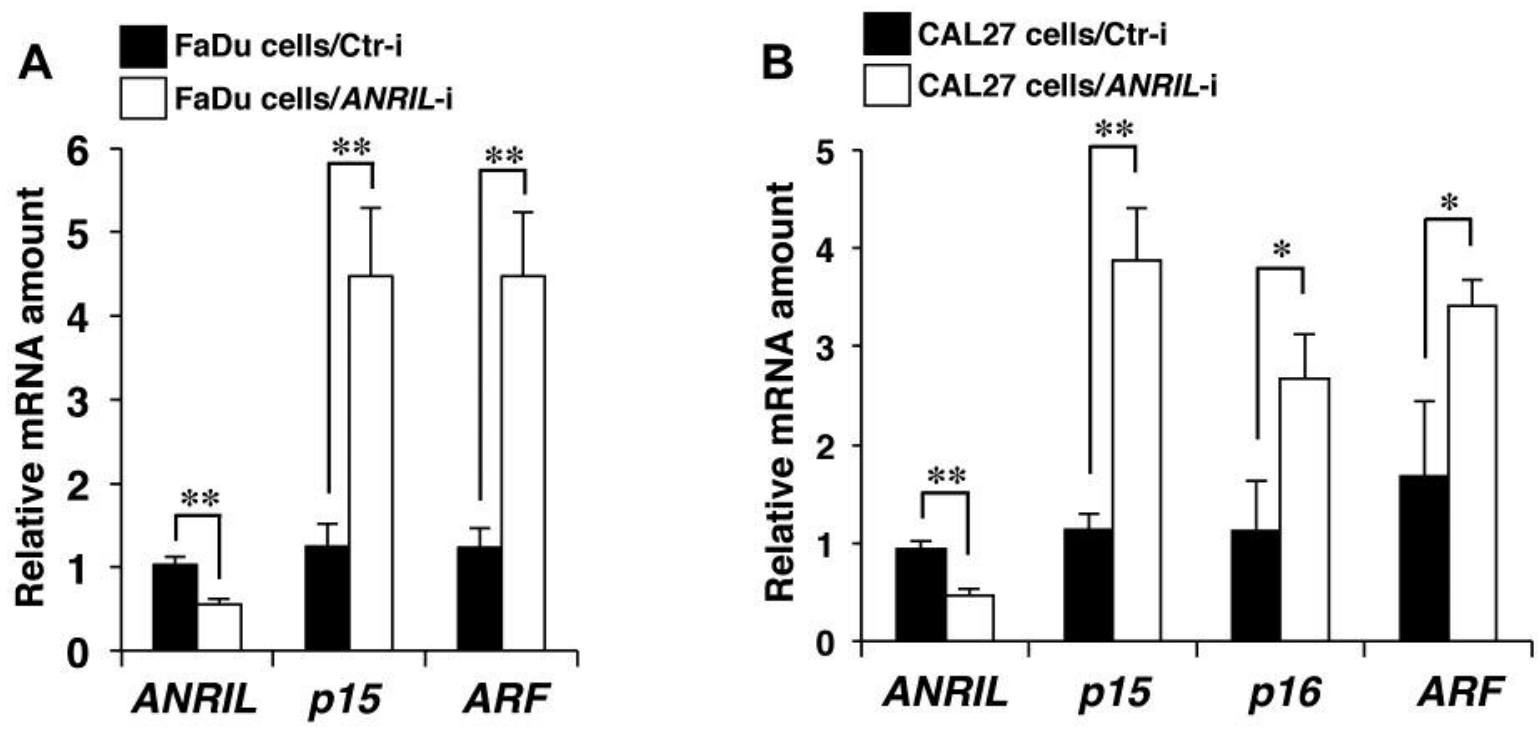

Figure 2. Depletion of ANRIL increases the expression of INK4 locus genes. A: FaDu cells were seeded at $5 \times 10^{5}$ cells/100mm dish and cultured for one day, transfected with either control siRNA (Ctr-i) or siRNA silencing ANRIL (ANRIL-i) and 72 hours later, were harvested to determine the $m R N A$ levels of ANRIL, $p 15$ and ARF by $q R T-P C R$. The results were expressed relative to the corresponding values for FaDu cells/Ctr-i. **p<0.01. B: qRT-PCR using CAL27 cells transfected with siRNA was performed and the data was analyzed as in (A). * $p<0.05, * * p<0.01$.

A

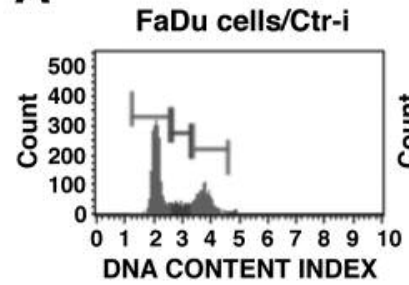

B

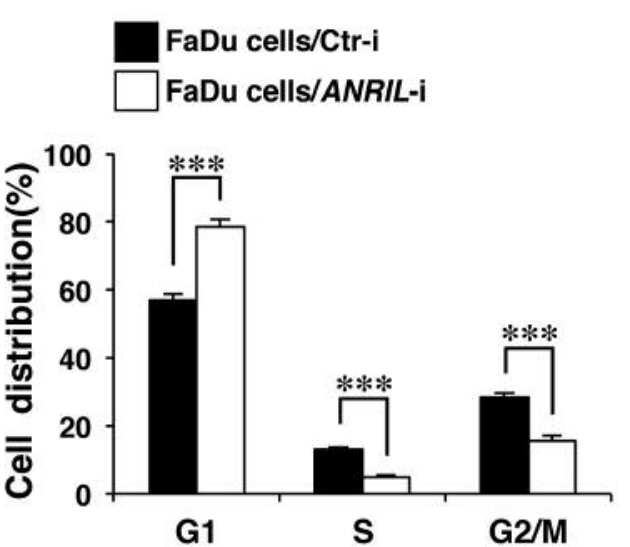

C
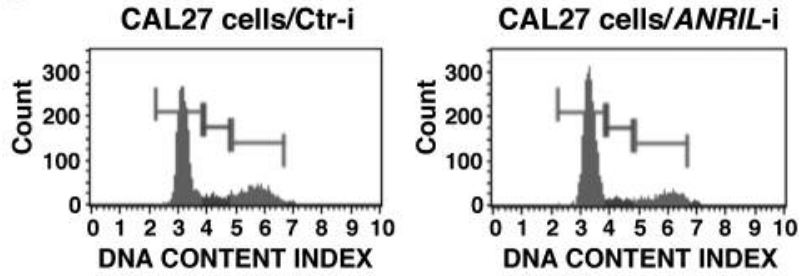

D

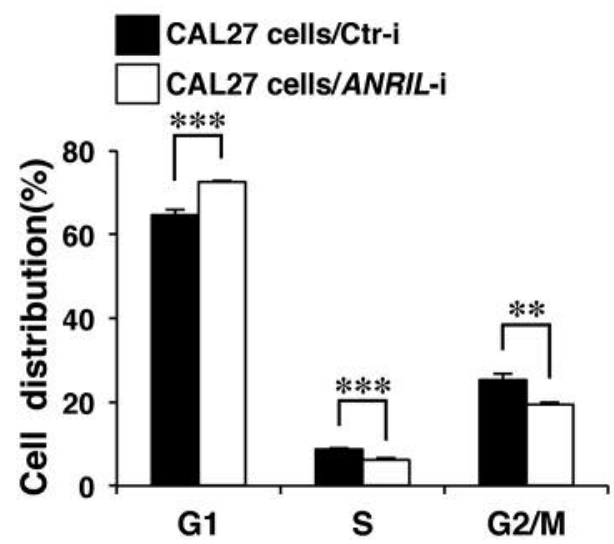

Figure 3. Depletion of ANRIL causes arrest in the $G_{1}$ phase of the cell cycle in both FaDu and CAL27 cells. A: At 72 h after transfection with either control siRNA (Ctr-i) or siRNA silencing ANRIL (ANRIL-i), FaDu cells were subjected to cell-cycle analysis by flow cytometry. The x-axis of histogram indicates propidium iodide staining intensity which is reflective of DNA content. The y-axis indicates cell count. B: The graph indicates percentages of FaDu cells in $G_{1}, S$ and $G_{2} / M$ phases. ${ }^{* *} p<0.001$. C: CAL27 cells transfected with siRNA were subjected to cell-cycle analysis as in (A). D: The graph indicates percentages of CAL27 cells in $G_{1}, S$ and $G_{2} / M$ phases. ${ }^{* *} p<0.01, *^{* *} p<0.001$. 
3 Zhang Y, Xiong Y and Yarbrough WG: ARF promotes MDM2 degradation and stabilizes p53: ARF-INK4a locus deletion impairs both the $\mathrm{Rb}$ and $\mathrm{p} 53$ tumor suppression pathways. Cell 92: 725-734, 1998. PMID: 9529249.

4 Sharpless NE: INK4a/ARF: a multifunctional tumor suppressor locus. Mutat Res 576: 22-38, 2005. PMID: 15878778. DOI: 10.1016/j.mrfmmm.2004.08.021

5 Kitagawa M, Kotake Y and Ohhata T: Long non-coding RNAs involved in cancer development and cell fate determination. Curr Drug Targets 13: 1616-1621, 2012. PMID: 22974399.

6 Pasmant E, Laurendeau I, Heron D, Vidaud M, Vidaud D and Bieche I: Characterization of a germ-line deletion, including the entire INK4/ARF locus, in a melanoma-neural system tumor family: identification of ANRIL, an antisense noncoding RNA whose expression coclusters with ARF. Cancer Res 67: 39633969, 2007. PMID: 17440112. DOI: 10.1158/0008-5472.CAN06-2004

7 Yap KL, Li S, Munoz-Cabello AM, Raguz S, Zeng L, Mujtaba S, Gil J, Walsh MJ and Zhou MM: Molecular interplay of the noncoding RNA ANRIL and methylated histone $\mathrm{H} 3$ lysine 27 by polycomb CBX7 in transcriptional silencing of INK4a. Mol Cell 38: 662-674, 2010. PMID: 20541999. DOI: 10.1016/j.molcel. 2010.03.021

8 Kotake Y, Nakagawa T, Kitagawa K, Suzuki S, Liu N, Kitagawa $\mathrm{M}$ and Xiong $\mathrm{Y}$ : Long non-coding RNA ANRIL is required for the PRC2 recruitment to and silencing of p15(INK4B) tumor suppressor gene. Oncogene 30: 1956-1962, 2011. PMID: 21151178. DOI: $10.1038 /$ onc 2010.568

9 Cao R, Tsukada Y and Zhang Y: Role of Bmi-1 and Ring1A in H2A ubiquitylation and Hox gene silencing. Mol Cell 20: 845854, 2005. PMID: 16359901. DOI: 10.1016/j.molcel.2005. 12.002

10 Wang H, Wang L, Erdjument-Bromage H, Vidal M, Tempst P, Jones RS and Zhang Y: Role of histone H2A ubiquitination in Polycomb silencing. Nature 431: 873-878, 2004. PMID: 15386022. DOI: 10.1038 /nature02985

11 Wang L, Brown JL, Cao R, Zhang Y, Kassis JA and Jones RS: Hierarchical recruitment of polycomb group silencing complexes. Mol Cell 14: 637-646, 2004. PMID: 15175158. DOI: 10.1016/j.molcel.2004.05.009

12 Congrains A, Kamide K, Katsuya T, Yasuda O, Oguro R, Yamamoto K, Ohishi M and Rakugi H: CVD-associated noncoding RNA, ANRIL, modulates expression of atherogenic pathways in VSMC. Biochem Biophys Res Commun 419: 612 616, 2012. PMID: 22382030. DOI: 10.1016/j.bbrc.2012.02.050

13 Sato K, Nakagawa H, Tajima A, Yoshida K and Inoue I: ANRIL is implicated in the regulation of nucleus and potential transcriptional target of E2F1. Oncol Rep 24: 701-707, 2010 PMID: 20664976.
14 Holdt LM, Hoffmann S, Sass K, Langenberger D, Scholz M, Krohn K, Finstermeier K, Stahringer A, Wilfert W, Beutner F, Gielen S, Schuler G, Gabel G, Bergert H, Bechmann I, Stadler $\mathrm{PF}$, Thiery $\mathrm{J}$ and Teupser D: Alu elements in ANRIL non-coding RNA at chromosome 9p21 modulate atherogenic cell functions through trans-regulation of gene networks. PLoS Genet 9: e1003588, 2013. PMID: 23861667. DOI: 10.1371/journal.pgen. 1003588

15 Naemura M, Murasaki C, Inoue Y, Okamoto H and Kotake Y: Long noncoding RNA ANRIL regulates proliferation of nonsmall cell lung cancer and cervical cancer cells. Anticancer Res 35: 5377-5382, 2015. PMID: 26408699.

16 Naemura M, Tsunoda T, Inoue $Y$, Okamoto $H$, Shirasawa $S$ and Kotake Y: ANRIL regulates the proliferation of human colorectal cancer cells in both two- and three-dimensional culture. Mol Cell Biochem 412: 141-146, 2016. PMID: 26708220. DOI: 10.1007/s11010-015-2618-5

$17 \mathrm{Li} \mathrm{Z,} \mathrm{Yu} \mathrm{X} \mathrm{and} \mathrm{Shen} \mathrm{J:} \mathrm{ANRIL:} \mathrm{a} \mathrm{pivotal} \mathrm{tumor} \mathrm{suppressor} \mathrm{long}$ non-coding RNA in human cancers. Tumour Biol 37: 5657-5661, 2016. PMID: 26753962. DOI: 10.1007/s13277-016-4808-5

18 Chai L, Yuan Y, Chen C, Zhou J and Wu Y: The role of long non-coding RNA ANRIL in the carcinogenesis of oral cancer by targeting miR-125a. Biomed Pharmacother 103: 38-45, 2018. PMID: 29635126. DOI: 10.1016/j.biopha.2018.01.105

19 Kotake Y, Cao R, Viatour P, Sage J, Zhang Y and Xiong Y: pRB family proteins are required for $\mathrm{H} 3 \mathrm{~K} 27$ trimethylation and Polycomb repression complexes binding to and silencing p16INK4alpha tumor suppressor gene. Genes Dev 21: 49-54, 2007. PMID: 17210787. DOI: 10.1101/gad.1499407

20 Kotake Y, Goto T, Naemura M, Inoue Y, Okamoto H and Tahara $\mathrm{K}$ : Long Noncoding RNA PANDA positively regulates proliferation of osteosarcoma cells. Anticancer Res 37: 81-85, 2017. PMID: 28011477. DOI: 10.21873/anticanres.11292

21 Zhang LM, Ju HY, Wu YT, Guo W, Mao L, Ma HL, Xia WY, $\mathrm{Hu}$ JZ and Ren GX: Long non-coding RNA ANRIL promotes tumorgenesis through regulation of FGFR1 expression by sponging miR-125a-3p in head and neck squamous cell carcinoma. Am J Cancer Res 8: 2296-2310, 2018. PMID: 30555745 . 